\title{
Anisotropic phase-map denoising using a regularized cost-function with complex-valued Markov-random-fields
}

\author{
Jesús Villa ${ }^{\mathrm{a}, *}$, Ramón Rodríguez-Vera ${ }^{\mathrm{b}}$, Juan Antonio Quiroga ${ }^{\mathrm{c}}$, Ismael de la Rosa ${ }^{\mathrm{a}}$, Efrén González ${ }^{\mathrm{a}}$

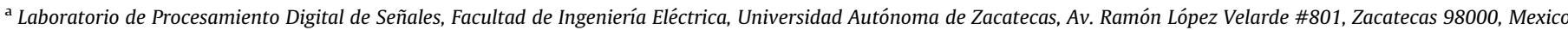 \\ ${ }^{\mathrm{b}}$ Centro de Investigaciones en Óptica A.C., León, Guanajuato 20036, Mexico

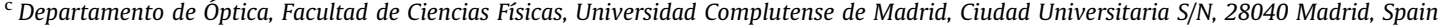

\section{A R T I C L E I N F O}

\section{Article history:}

Received 29 October 2009

Received in revised form

29 January 2010

Accepted 6 February 2010

Available online 26 February 2010

Keywords:

Regularization

Phase-map filtering

\begin{abstract}
A B S T R A C T
In our recently reported work [1] (Villa et al., 2009) we derived a regularized quadratic-cost function, which includes fringe orientation information, for denosing fringe pattern images. In this work we adopt such idea for denoising wrapped phase-maps. We use a regularized cost-function that uses complex-valued Markov random fields (CMRFs) with orientation information of the filtering direction along isophase lines. The advantage of using an anisotropic filter along isophase lines is that phase and noise can be properly separated while $2 \pi$ phase jumps are preserved even in high frequency zones. Apart from its robustness, the outstanding advantage of our method is its minimal computational effort. We present some results processing simulated and real phase-maps.
\end{abstract}

(c) 2010 Elsevier Ltd. All rights reserved.

\section{Introduction}

In fringe pattern analysis the aim is to recover the phase that modulates the fringes. The phase is important because it is continuously related with a physical quantity, such as wave fronts, topography, etc. However, most fringe demodulation techniques give the phase wrapped, so that the continuous phase must be obtained by means of the well-known unwrapping process. Phase unwrapping is an important and well-known task in several areas [2], such as speckle interferometry [3], holographic inteferometry [4], profilometry [5] and remote sensing $[6,7]$.

To briefly describe the unwrapping problem we define the continuous phase field as $\phi_{r}$, where $r=(i, j)$ is the vector of image coordinates in a regular lattice $L$. The wrapped phase (i.e. the observed phase) is then defined as $\alpha_{r}=W\left[\phi_{r}\right]$, where $W$ is the wrapping operator such that $\alpha_{r} \in[-\pi, \pi)$.

The problem of recovering the continuous phase $\phi_{r}$ from $\alpha_{r}$ may be solved if we have prior information about $\phi_{r}$. For example if we know that $\phi_{r}$ varies slowly from pixel to pixel, the relation

$W\left[\nabla \alpha_{r}\right]=\nabla \phi_{r}$

holds. In other words, the wrapped gradient of the wrapped phase equals the gradient of the continuous phase. Consequently, the recovery of the continuous phase without ambiguities is possible

\footnotetext{
* Corresponding author.

E-mail address: jvillah@uaz.edu.mx (J. Villa).
}

by summation of the wrapped gradient of $\alpha_{r}$. Unfortunately, in many practical situations the observed phase $\alpha_{r}$ is affected by noise, so that it is not guaranteed to satisfy relation (1), and ambiguities may arise.

No matter which kind of technique is used, a filtering process to preserve phase details becomes a key step for an easier and more reliable phase unwrapping.

The widely used denoising techniques for images, for example convolution techniques or Fourier techniques generally produces over-smoothed images that may smear out discontinuities of wrapped phase fields, particularly in high frequency zones. Some denoising techniques have been developed for improving the phase unwrapping [3,4], for example we can find in the work by Kemao et al. [8] a comparative analysis of some methods. Recently, a more effective point of view for filtering wrapped phase maps has been pointed out by Tang et al. [9] who use sine/cosine filtering with windows along tangent directions of isophase contours of ESPI (electronic speckle pattern interferometry) phase maps. Even more recently, the same author proposed the oriented-couple partial differential equations [10] for denoising wrapped phase patterns. In both cases $[9,10]$ the key idea is the filtering along isophase lines (i.e. along the orthogonal direction of phase gradient). This strategy have two big advantages: first, noise can be easily removed because phase information and noise are conveniently separated in the frequency domain, and second, discontinuities of the wrapped phase map are preserved, specially in high frequency zones.

Since the last three decades regularization methods have been widely used for image processing [11-15] which have 
demonstrated to be a powerful tool for this purpose. The main idea of regularization methods is to reconstruct a degraded image by introducing prior information or assumptions about the structure of the original image, this information may be for example the smoothness of the image by means of an MRF (Markov random field) model. An example of the application of regularization methods for phase-maps denoising is the interesting work by Ferraiuolo and Poggi [6] who describe a Bayesian filtering technique for SAR (synthetic aperture radar) interferometric phase-fields, however the Ferraiuolo's technique does not use orientation information but uses a two-component MRF prior model: the first component penalizes residues, while the second one penalizes discontinuities. In our previously reported work [1], using the Bayesian approach, we derived a quadratic costfunction with MRFs that includes fringe orientation information to denoise fringe pattern images. In this paper we propose to follow the same philosophy to denoise wrapped phase-maps using a regularized quadratic cost-function with complex-valued Markov random fields (CMRFs) as prior models, including orientation information of isophase lines which permit removing the noise only along such lines. As will be shown, the phase-map denoising is carried out in a robust and simple manner owing that eliminates noise preserving $2 \pi$ phase jumps with a minimum computational effort.

This paper is organized in the following way. In Section 2 we describe the proposed method deriving a regularized costfunction with CMRFs. In Section 3 we describe the adopted technique for estimating the orientation field of isophase lines. In Section 3 we present some experimental results with simulated and real phase-maps. Finally, in Section 4 we give the conclusions.

\section{Bayesian phase-map denoising}

Considering the problem of denoising wrapped phase-maps the first drawback we found is that, owing to $2 \pi$ phase jumps of $\alpha_{r}$, direct application of any kind of filter is not always a proper procedure to solve it. For example, the application of a simple mean filter may smear out the phase jumps. In order to avoid this drawback we carry out the phase filtering in the following way. We define the complex function

$g_{r}=e^{i \alpha_{r}}$,

where $i=\sqrt{-1}$. As both imaginary and real parts of $g_{r}$ are continuous functions, we can properly apply a filter over $g_{r}$, and the argument of the obtained complex signal will contain the denoised phase-map.

In order for this paper to be self-contained we describe in the following subsections the Bayesian approach for isotropic and anisotropic denoising already reported in [1].

\subsection{Isotropic denoising}

We can model the observed complex image that contains the phase information corrupted by noise as $g_{r}=f_{r}+n_{r}$, where $f_{r}$ and $n_{r}$ represent the noiseless image and the aleatory noise with a given probability distribution, respectively. The Bayesian approach to regularize this kind of problem requires prior knowledge of $f$. The estimated signal may be obtained as the minimizer (the maximum a posteriori estimator) of an energy functional of the form [1]

$U\left(f_{r}\right)=\sum_{r \in L} \Phi\left(f_{r}-g_{r}\right)+\mu \sum_{C} V_{c}\left(f_{r}\right)$.

Parameter $\mu$ is a positive number that depends on the noise variance, which controls the compromise between the degree of

regularization and its closeness with the observed data. Note that we are dealing with complex-valued potential functions.

The first term in (3), called data term, establishes that the reconstruction of $f_{r}$ must be consistent with the observed data. The second term is the well-known regularization term that imposes a penalty for violating the a priori assumptions. In order to derive a cost function for phase denoising we may assume the noise is Gaussian and select a CMRF with a neighborhood system $C$ that corresponds to horizontal, vertical and diagonal firstneighbor elements, such that

$U_{A}\left(f_{r}\right)=\sum_{r \in L}\left[f_{r}-g_{r}\right]^{2}+\mu \sum_{C \in L}\left[f_{r}-f_{s}\right]^{2}$.

The first term imposes the estimated field to be close to the data in the sense of least-squares. On the other hand, the second term imposes the estimated field to minimize square differences along horizontal, vertical and diagonal directions. Fig. 1(a) shows the involved cliques around pixel $r$.

Introducing the image coordinates we can rewrite Eq. (4) as

$U_{A}\left(f_{i, j}\right)=\sum_{(i, j) \in L}\left\{\left[f_{i, j}-g_{i, j}\right]^{2}+\mu\left[C_{1}+C_{2}+C_{3}+C_{4}\right]\right\}$,

where

$C_{1}=\left[f_{i, j}-f_{i-1, j}\right]^{2}+\left[f_{i, j}-f_{i+1, j}\right]^{2}$,

$C_{2}=\left[f_{i, j}-f_{i, j-1}\right]^{2}+\left[f_{i, j}-f_{i, j+1}\right]^{2}$,

$C_{3}=\left[f_{i, j}-f_{i-1, j-1}\right]^{2}+\left[f_{i, j}-f_{i+1, j+1}\right]^{2}$,

$C_{4}=\left[f_{i, j}-f_{i+1, j-1}\right]^{2}+\left[f_{i, j}-f_{i-1, j+1}\right]^{2}$

The filtered image $f_{i, j}$ may be determined by setting

$\frac{\partial U_{A}}{\partial f_{i, j}}=0$,

which give us the following linear system:

$2\left[f_{i, j}-g_{i, j}\right]-2 \mu\left[f_{i-1, j}-2 f_{i, j}+f_{i+1, j}\right]-2 \mu\left[f_{i, j-1}-2 f_{i, j}+f_{i, j+1}\right]-2 \mu\left[f_{i-1, j-1}\right.$

$\left.-2 f_{i, j}+f_{i+1, j+1}\right]-2 \mu\left[f_{i+1, j-1}-2 f_{i, j}+f_{i-1, j+1}\right]=0$.

The estimator $\hat{f}_{\text {MAP }}$ of this cost-function will represent a low-pass filtered image. The filter that represents this cost-function, however, is an isotropic one, this means that it may smear out phase jumps of the wrapped phase.

\subsection{Anisotropic denoising}

As mentioned above, a better strategy for denoising wrapped phase-maps is by means of an anisotropic filter that work along isophase lines. Fig. 2 shows an example of a wrapped phase-map

a

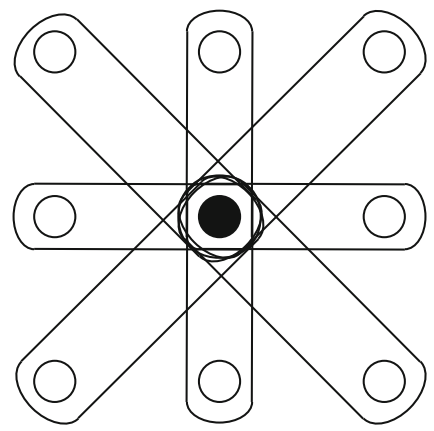

Fig. 1. Involved cliques around pixel $r$ (black circle). (a) Using cost-function (4),

(b) using cost-function (10). b

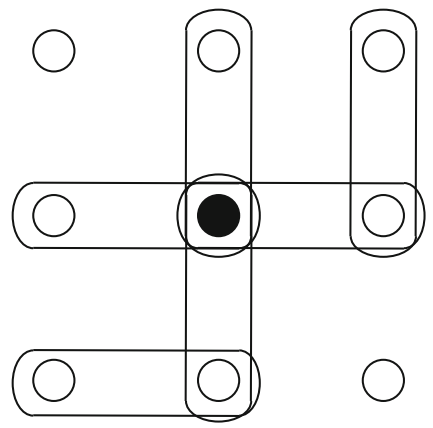


a

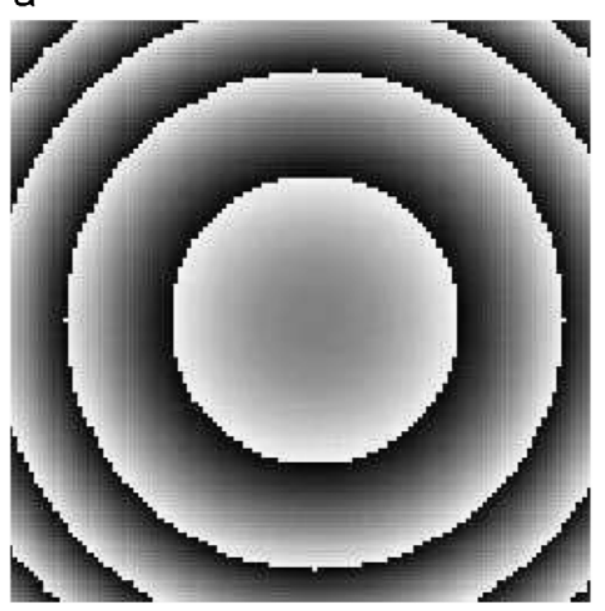

b

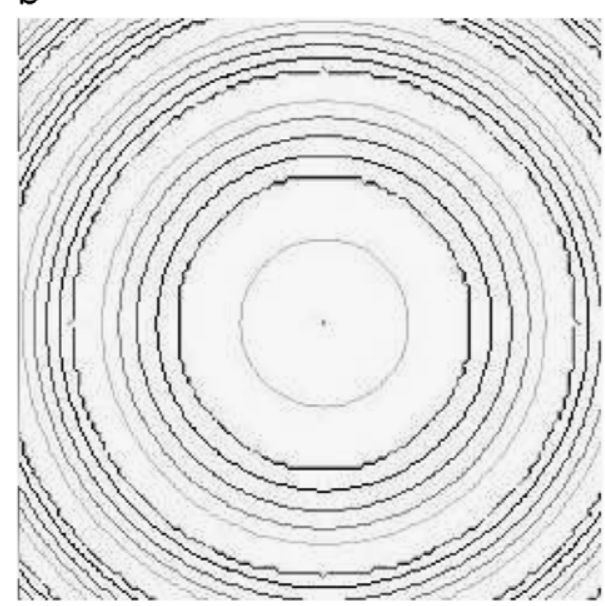

Fig. 2. (a) Gray level codification of a wrapped phase-map. (b) Its contour map of isophase lines.

and its corresponding contour map of isophase lines. As phase is constant along these lines, applying an oriented filter along them allows to properly separate noise from signal and reduces oversmoothing phase information along gradient direction that causes smearing out $2 \pi$ phase jumps.

Introducing the angle $\theta_{r}$ which represents the orientation field along isophase contours, we may define the directional derivative of $f_{r}$ along the unit vector $\left[\cos \theta_{r}, \sin \theta_{r}\right]$ as

$D_{r}=\left(f_{r}-f_{h}\right) \cos \theta_{r}+\left(f_{r}-f_{v}\right) \sin \theta_{r}$,

where $h$ and $v$ represent first-neighbors of $r$ along horizontal and vertical directions respectively.

Now, defining a CMRF with a neighborhood system that corresponds to horizontal and vertical elements as depicted in Fig. 1(b), we may propose the following cost-function which represents an anisotropic low-pass filter along isophase lines:

$U_{B}\left(f_{r}\right)=\sum_{r \in L}\left[f_{r}-g_{r}\right]^{2}+\mu \sum_{C \in L}\left[\left(f_{r}-f_{h}\right) \cos \theta_{r}+\left(f_{r}-f_{v}\right) \sin \theta_{r}\right]^{2}$.

Introducing the image coordinates we can rewrite it as

$U_{B}\left(f_{i, j}\right)=\sum_{(i, j) \in L}\left\{\left[f_{i, j}-g_{i, j}\right]^{2}+\mu\left[D_{1}^{2}+D_{2}^{2}+D_{3}^{2}\right]\right\}$,

where

$D_{1}=\left(f_{i, j}-f_{i-1, j}\right) c_{i, j}+\left(f_{i, j}-f_{i, j-1}\right) s_{i, j}$,

$D_{2}=\left(f_{i+1, j}-f_{i, j}\right) c_{i+1, j}+\left(f_{i+1, j}-f_{i+1, j-1}\right) s_{i+1, j}$,

$D_{3}=\left(f_{i, j+1}-f_{i-1, j+1}\right) c_{i, j+1}+\left(f_{i, j+1}-f_{i, j}\right) s_{i, j+1}$,

$c_{i, j}=\cos \theta_{i, j}$ and $s_{i, j}=\sin \theta_{i, j}$.

We set $\partial U_{B} / \partial f_{i, j}=0$ such that $f_{i, j}$ can be obtained from the linear system

$$
\begin{aligned}
& 2\left[f_{i, j}-g_{i, j}\right]+2 \mu\left[\left(f_{i, j}-f_{i-1, j}\right) c_{i, j}+\left(f_{i, j}-f_{i, j-1}\right) s_{i, j}\right]\left(c_{i, j}+s_{i, j}\right) \\
& \quad-2 \mu\left[\left(f_{i+1, j}-f_{i, j}\right) c_{i+1, j}+\left(f_{i+1, j}-f_{i+1, j-1}\right) s_{i+1, j}\right] c_{i+1, j} \\
& \quad-2 \mu\left[\left(f_{i, j+1}-f_{i-1, j+1}\right) c_{i, j+1}+\left(f_{i, j+1}-f_{i, j}\right) s_{i, j+1}\right] s_{i, j+1}=0 .
\end{aligned}
$$

Optimization of either $U_{A}$ or $U_{B}$ can be carried out using iterative methods [16]; particularly, in our experiments we used the gradient-descent algorithm:

$f^{k+1}=f^{k}-\lambda \frac{\partial U^{k}}{\partial f}$

where $\lambda$ is a positive constant.

\section{Computation of the orientation field of isophase lines}

A key step before implementing the anisotropic filter represented by cost-function (11) is the determination of the orientation field of isophase lines. One way to determine it is by means of the direct computation of phase gradient however, owing to noise and $2 \pi$ phase jumps of $\alpha_{r}$, the computed field may present errors and inconsistencies. Knowing that isophase lines of a phase map are parallel to its associated fringe contours, a better way to compute $\theta_{r}$ is just by computing the fringe orientation map of the real part of $g_{r}$. In this work we adopted the technique reported by Yang et al. [17] to compute this fringe orientation map.

Here we describe the method to compute $\theta_{i, j}$. First we define the fringe pattern

$I_{i, j}=\operatorname{Re}\left\{g_{i, j}\right\}=\cos \left(\alpha_{i, j}\right)$.

To obtain the fringe orientation map of $I_{i, j}$, we compute central differences along horizontal, vertical and diagonal directions:

$d_{i, j}^{0}=\left|I_{i-1, j}-I_{i+1, j}\right| \sqrt{2}, \quad d_{i, j}^{45}=\left|I_{i-1, j+1}-I_{i+1, j-1}\right|$,

$d_{i, j}^{90}=\left|I_{i, j-1}-I_{i, j+1}\right| \sqrt{2}, \quad d_{i, j}^{135}=\left|I_{i-1, j-1}-I_{i+1, j+1}\right|$.

Factor $\sqrt{2}$ is used in horizontal and vertical differences for consistency with respect to diagonal distances. In order to minimize the effects of noise the authors of this method proposed an accumulating strategy by sums of these differences in square regions $\Gamma \in L$ with center $(i, j)$ such that the orientation is obtained with

$\left.\beta_{i, j}=\frac{1}{2} \arctan \left(\sum_{(k, l) \in \Gamma} d_{k, l}^{45}-\sum_{(k, l) \in \Gamma} d_{k, l}^{135}, \sum_{(k, l) \in \Gamma} d_{k, l}^{0}-\sum_{(k, l}\right) \in \Gamma d_{k, l}^{90}\right)$,

where $\arctan (\cdot, \cdot) \in[-\pi, \pi)$. Note that $\beta_{i, j}$ represents an orthogonal direction to $\theta_{i, j}$, however we can compute $\theta_{i . j}$ just by adding $\pi / 2$.

Fig. 3 sketches the relation between $\beta$ and $\theta$ trough vectors $\mathbf{v}=[\cos \beta, \sin \beta]$ and $\mathbf{u}=[\cos \theta, \sin \theta]$ respectively. Note that $\mathbf{v}$ is parallel to phase gradient.

\section{Experimental results}

In this section we present the results processing two simulated phase-maps and two experimentally obtained ESPI phase-maps. In the two simulations we compare the results applying our proposal and the WFT filtering technique [18]. In all our 
experiments we applied the optimization technique described in Eq. (14) with 25 iterations and using $\lambda=0.015$. The algorithms were implemented using Matlab in a $2.66 \mathrm{GHz}$ Pentium D based computer. The problem to determine the so-called regularization parameter $\mu$ has been widely discussed in the literature. Unfortunately, there are no explicit formula or method to determine it owing that it depends on the magnitude of the observed data, noise, etc. In our case, this parameter controls the wide-band of the filters (the higher the value of $\mu$ the narrower the wide-band). Many times this parameter is heuristically determined according to the problem and characteristics of the image to be processed. For this application it is determined according to the noise level but, fortunately, does not have to be abruptly changed for different cases of a given class of fringe pattern images.

The first experiment was realized processing a simulated noisy phase-map which is shown in Fig. 4(a). In this case we added Gaussian noise with zero-mean and 0.6 standard-deviation. Fig. 4(b) shows the result using cost-function (5) (isotropic denoising) with $\mu=6$. We can observe in Fig. 4(c) a better result applying the anisotropic filtering using cost-function (11) with $\mu=15$. In Fig. 4(d) we show the result applying the WFT technique technique has a better performance its big drawback is the long processing time as will be presented in Table 1. In Fig. 5 we show the results of the second experiment with a simulation. Fig. 5(a) is the original image with zero-mean and 0.7 standard-deviation Gaussian noise. Using cost-function (5) with $\mu=8$ we obtained the result shown in Fig. 5(b). Fig. 5(c) is the result using cost-

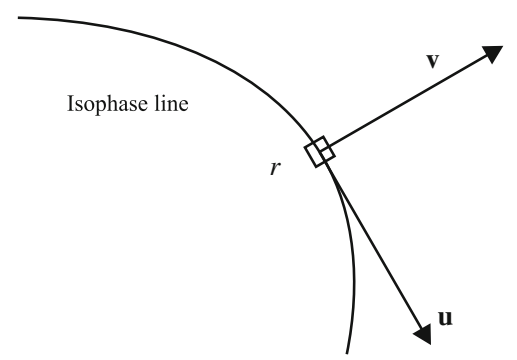

Fig. 3. Isophase line and the relation between $\beta$ and $\theta$ through vectors $\mathbf{v}=[\cos \beta, \sin \beta]$ and $\mathbf{u}=[\cos \theta, \sin \theta]$. reported by Kemao et al. [18]; although visually the WFT

function (11) with $\mu=15$. Fig. 5(d) shows the result applying the WFT technique.

The following two experiments were realized over experimentally obtained phase-maps. Fig. 6(a) shows the phase-map image of the third experiment. In this case it was obtained with a Michelson interferometer to measure temperature distribution of a flame. The result obtained using cost-function (5) with $\mu=5$ is shown in Fig. 6(b). Using cost-function (11) with $\mu=12$ we obtained the filtered image of Fig. 6(c). Finally, Fig. 7(a) shows the phase-map image of the fourth experiment. It was obtained using ESPI to measure displacement applying a tension charge over a plate of composite material. We can observe the results in Figs. 7(b) and (c) using cost-function (5) with $\mu=10$, and costfunction (11) with $\mu=15$ respectively.

It is important to remark that the presence of phasesingularities may cause a low-performance of the anisotropic filter owing that the orientation cannot be properly computed. See for example the central hole of Fig. 7(a) where valid information does not exist. However, to carry out a successful phase unwrapping (after denoising), we think this information may be isolated by means of a process to recover a ROI (region of interest) map. Finally, this information may be considered in the phase unwrapping.

In the simulated experiments we carried out a quantitative evaluation of the filters computing the normalized mean-square error (NMSE) [19], which is defined as

$\varepsilon=\frac{\sum|\alpha-\hat{\alpha}|^{2}}{\sum|\alpha|^{2}}$

where $\alpha$ and $\hat{\alpha}$ represent the noiseless phase-map and the estimated phase-map respectively. Table 1 resumes the performance of the filters in the experiments.

Table 1

Performance of isotropic, anisotropic and Windowed Fourier filters represented by $U_{A}, U_{B}$ and WFT respectively.

\begin{tabular}{|c|c|c|c|c|c|c|c|c|c|}
\hline \multirow[t]{2}{*}{ Figure } & \multirow[t]{2}{*}{ Size } & \multicolumn{3}{|c|}{ Processing time $(\mathrm{s})$} & \multicolumn{3}{|c|}{$\operatorname{NMSE}(\varepsilon)$} & \multicolumn{2}{|l|}{$\mu$} \\
\hline & & $U_{A}$ & $U_{B}$ & WFT & $U_{A}$ & $U_{B}$ & WFT & $U_{A}$ & $U_{B}$ \\
\hline 4(a) & $300 \times 150$ & 1.3 & 1.5 & 168 & 0.2133 & 0.0724 & 0.1381 & 6 & 15 \\
\hline $5(a)$ & $400 \times 400$ & 5.1 & 5.7 & 320 & 0.0934 & 0.0637 & 0.0557 & 8 & 15 \\
\hline 6(a) & $450 \times 560$ & 7.3 & 8.2 & - & - & - & - & 5 & 12 \\
\hline 7(a) & $400 \times 480$ & 5.7 & 6.4 & - & - & - & - & 10 & 15 \\
\hline
\end{tabular}
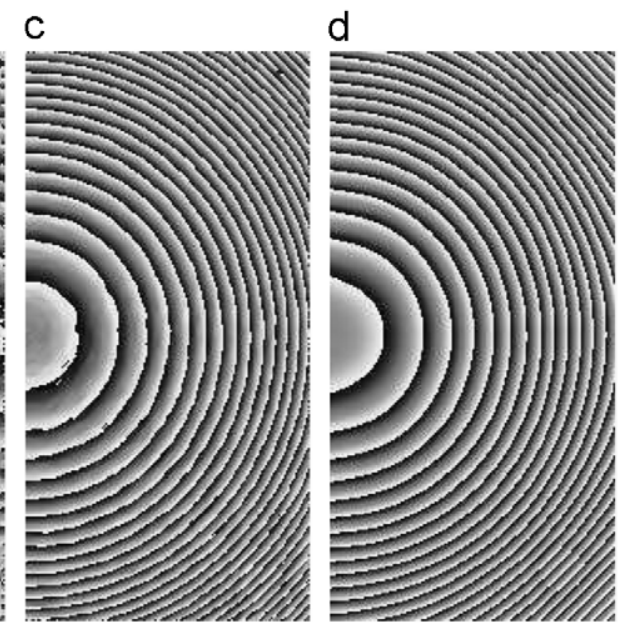

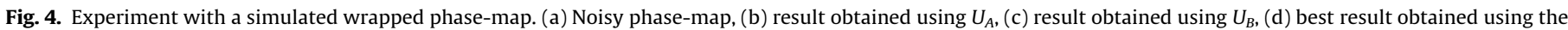

WFT technique.

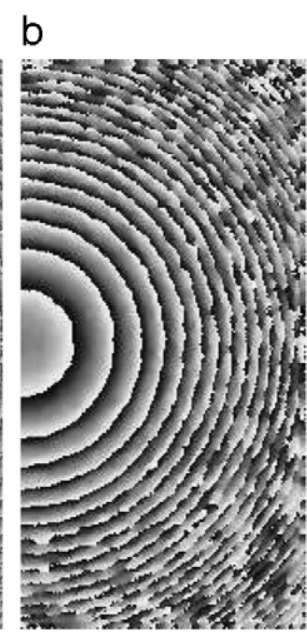


a

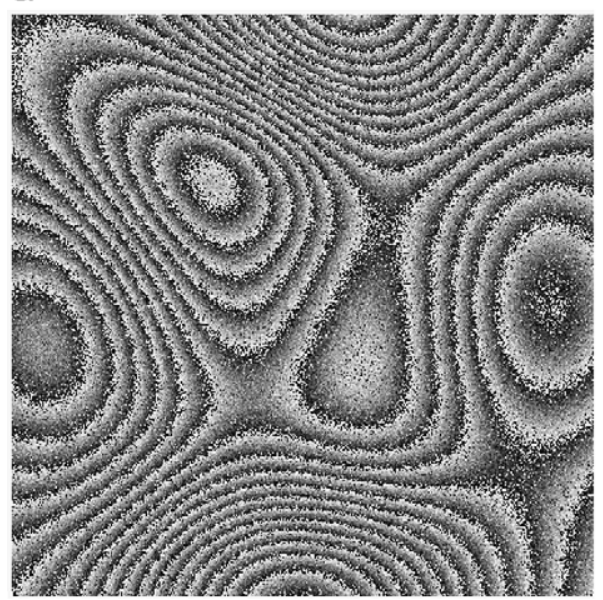

C

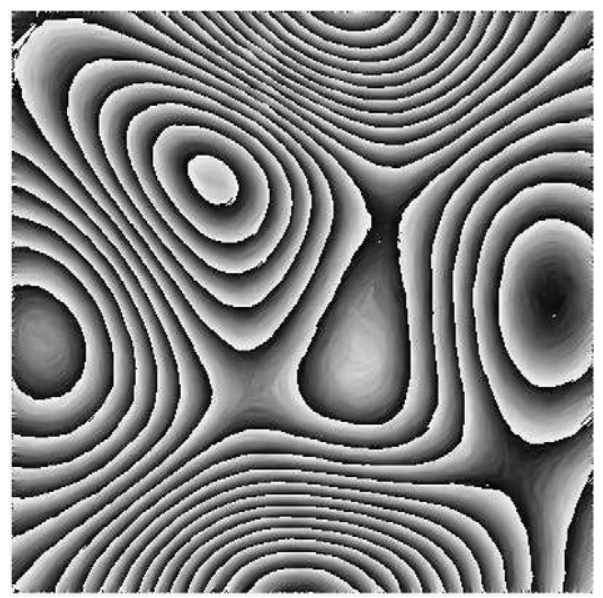

b

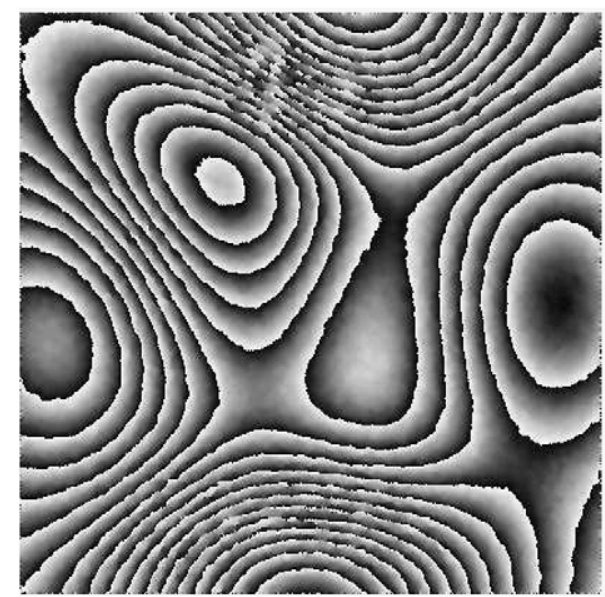

d

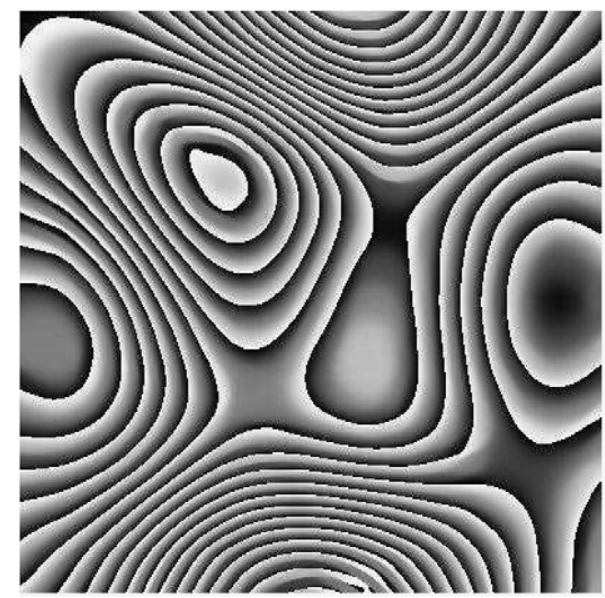

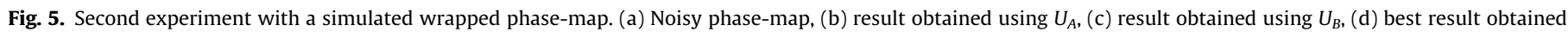
using the WFT technique.

From the above presented experiments we obviously deduce that an anisotropic filter has better performance than an isotropic one. Particularly, by means of cost-function (11) noise is effectively suppressed while phase-jumps are perfectly preserved even in the highest frequency zones. Analyzing Eq. (13) we may deduce that if $g_{i, j}$ is a continuous function the linear system is well conditioned and the optimization algorithm converges with few iterations. These are two attractive aspects of our denoising method because it can be easily implemented and requires a short processing time compared with Tang et al.'s method reported in [10].

In some cases, owing to erroneous orientation-estimations and spatial variation of filtering orientation, the results obtained applying our filtering method present wavy structures in lowfrequency zones, which requires a further filtering process. In these situations we just applied the isotropic filter represented by cost-function (5) using a small value of parameter $\mu$ (typically $0.5<\mu<1.0$ ) in order to preserve $2 \pi$ phase jumps.

Owing that the filter works along the orthogonal direction to phase gradient which means that local frequencies of signal and noise are well separated, in the four experiments we can see that our anisotropic filter has superior performance in high frequency zones. Reviewing already reported anisotropic filters (for example the work by Tang et al. [10]) we can see a similar performance against noise compared with our technique, however, the work reported in [10] requires a strong computational effort and a long processing time. Also, the use of other kind of filters adapted to wrapped phase-map denoising, such as the works by Kemao et al. [18] and Goldstein et al. [20], present similar disadvantages. For example, using the technique by Kemao is difficult to control its parameters to obtain good results. On the other hand, the two main attractiveness of our proposal are: (1) the minimal computational effort with a well-theoretical foundation. (2) The short processing time. As far as we know, already reported anisotropic filters for phase map denoising (even other kind of filters) do not join these two advantages.

\section{Conclusions}

It is evident that denoising wrapped phase-maps plays a key role for a successful phase unwrapping. In all presented experiments we can observe the superior performance of an anisotropic filter for denoising wrapped phase-maps. Unlike isotropic filters, anisotropic filters may properly preserve $2 \pi$ phase jumps even in high frequency zones. Particularly, we have demonstrated that the proposed regularization technique with CMRFs is a powerful alternative for this purpose in two aspects. First, experimental results show that the outstanding performance against noise. Second, apart from the robustness, another outstanding advantage of the proposed technique is its minimal computational effort compared with some already reported techniques. 
a

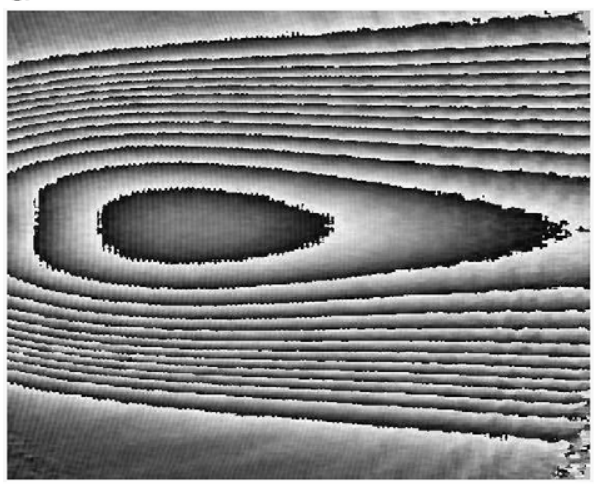

b

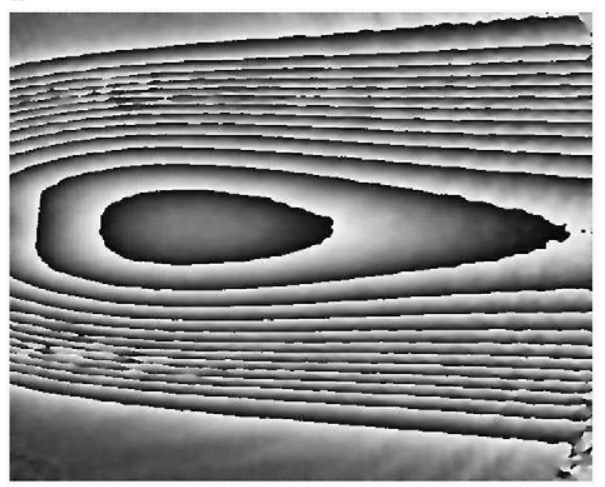

C

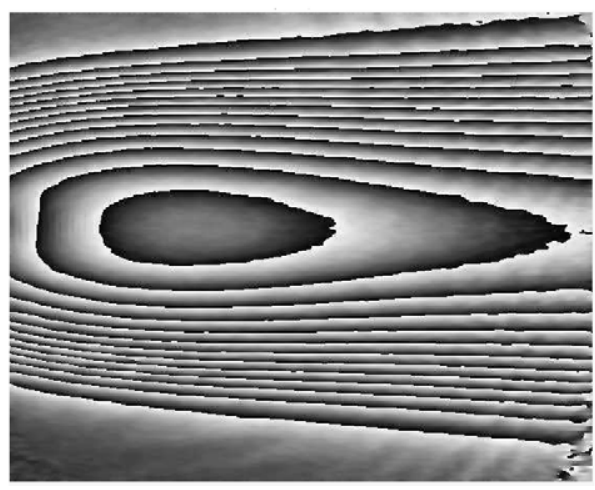

Fig. 6. (a) Experimentally obtained ESPI phase-map. (b) Result obtained using $U_{A}$, (c) result obtained using cost-function $U_{B}$.

a

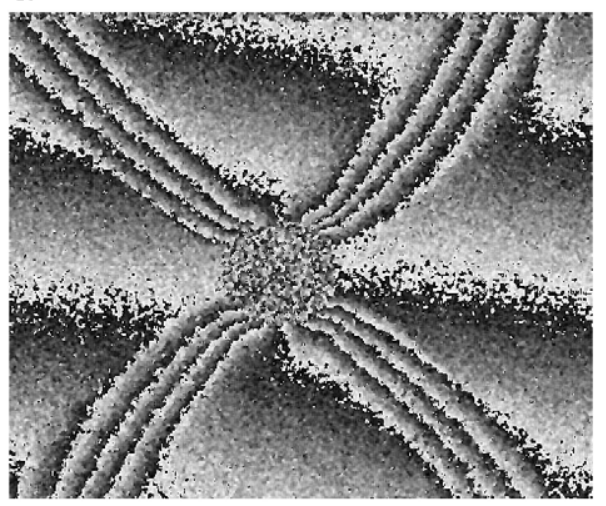

b

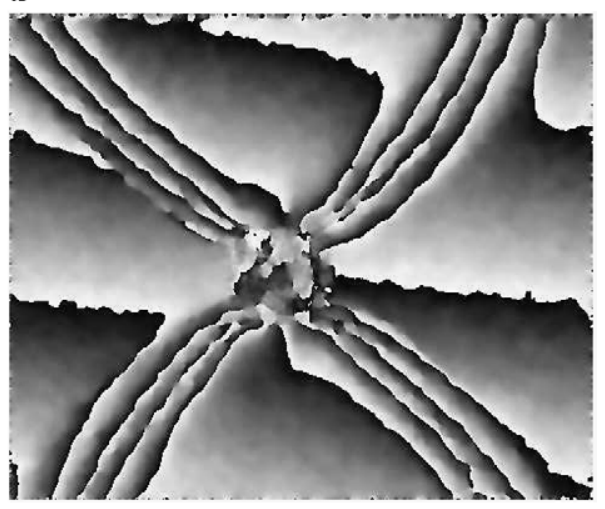

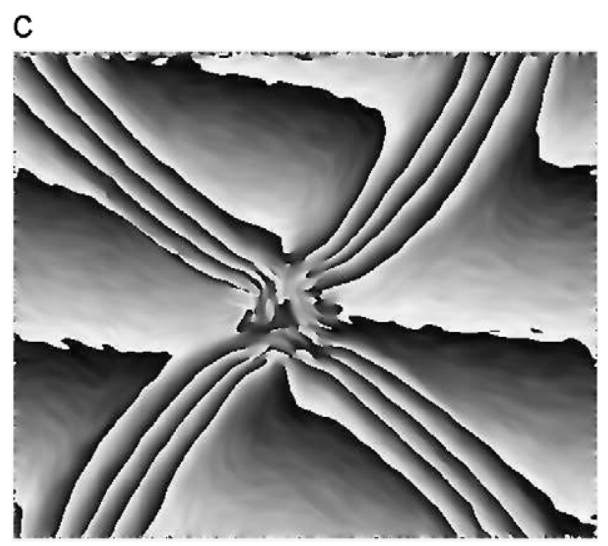

Fig. 7. (a) Experimentally obtained ESPI phase-map. (b) Result obtained using $U_{A}$, (c) result obtained using $U_{B}$. 


\section{Acknowledgments}

We acknowledge the partial support for the realization of this work to the Secretaría de Educación Pública of México through the project PIFI-2007, and the Ministerio de Ciencia y Tecnología of Spain trough the project DPI2005-03891.

\section{References}

[1] Villa J, Antonio Quiroga J, de la Rosa I. Regularized quadratic cost function for oriented fringe-pattern filtering. Opt Lett 2009;34(11):1741-3.

[2] Ghiglia DG, Pritt MD. Two-dimensional phase-unwrapping. New York, N.Y.: Johon Wiley \& Sons; 1998.

[3] Aebischer HA, Waldner S. A simple and effective method for filtering speckleinterferometric phase fringe patterns. Opt Commun 1999;162:205-10.

[4] Palacios F, Goncalves E, Ricardo J, Valin JL. Adaptive filter to improve the performance of phase-unwrapping in digital holography. Opt Commun 2004; 238:245-51.

[5] Takeda M, Ina H, Kobayashi S. Fourier transform method of fringe pattern analysis for computer based topography and interferometry. J Opt Soc Am 1982;72:156-60

[6] Ferraiuolo G, Poggi G. A Bayesian filtering technique for SAR interferometric phase fields. IEEE Trans Image Process 2004;13(10):1368-78.

[7] Yu Q, Yang X, Fu S, Liu X, Sun X. An adaptive contoured window filter for interferometric synthetic aperture radar. IEEE Geosci Remote Sensing Lett 2007;4(1):23-6.
[8] Kemao Q, Nam LTH, Feng L, Soon SH. Comparative analysis on some filters for wrapped phase maps. Appl Opt 2007;46(30):7412-8.

[9] Tang C, Wang W, Yan H, Gu X. Tangent least-squares fitting filtering method for electrical speckle pattern interferometry phase fringe patterns. Appl Opt 2007;46(15):2907-13.

[10] Tang C, Han L, Ren H, Gao T, Wang Z, Tang K. The oriented-couple partial differential equations for filtering in wrapped phase patterns. Opt Express 2009;17(7):5606-17.

[11] Besag JE. Spatial interaction and the statistical analysis of lattice systems. J R Stat Soc Ser B 1974;B-36:192-236.

[12] Geman S, Geman D. Stochastic relaxation, Gibbs distribution, and the Bayesian restoration of images. IEEE Trans Pattern Anal Machine Intell 1984 PAMI-6:721-41.

[13] Rivera M, Marroquin JL. Efficient half-quadratic regularization with granularity control. Image Vision Comput 2003;21:345-57.

[14] Derin H, Elliot H, Cristi R, Geman D. Bayes smoothing algorithms for segmentation. IEEE Trans Pattern Anal Mach Intell 1984;PAMI-6:707-20.

[15] Bouman C, Sauer K. A generalized gaussian image model for edge-preserving MAP estimation. IEEE Trans Nucl Sci 1992;99(4):1144-52.

[16] Golub GH, Van Loan CF. Matrix computations. Baltimore, Maryland: Johns Hopkins University Press; 1990.

[17] Yang X, Yu Q, Fu S. An algorithm for estimating both fringe orientation and fringe density. Opt Commun 2007;274:286-92.

[18] Kemao Q. Two-dimensional windowed Fourier transform for fringe pattern analysis: principles, applications and implementations. Opt Lasers Eng 2007; 45:304-17.

[19] Fienup JR. Invariant error metrics for image reconstruction. Appl Opt 1997; 36:8352-7.

[20] Goldstein RM, Werner CL. Radar interferogram filtering for geophysical applications. Geophys Res Lett 1998;25(21):4035-8. 